\title{
Value of Sono-Elastography in Differentiation between Benign and Malignant Thyroid Nodules
}

\author{
AMANY M.R. ABDEL-AZIZ, M.D.; EMAN A.F. DARWISH, M.D. and SARA M.M. MAHMOUD, M.Sc. \\ The Department of Diagnostic Radiology, Faculty of Medicine, Ain Shams University
}

\begin{abstract}
Background: The evaluation of thyroid nodules poses a challenge. Gray-scale ultrasound is excellent for the detection and characterization of thyroid nodules, but the accuracy for the differentiation between benign and malignant lesions based on single criteria is low.
\end{abstract}

Aim of Study: To assess the diagnostic value of elastography in the evaluation of thyroid nodules.

Patients and Methods: This study was performed on 25 thyroid nodules in 25 adult patients ( $>18$ years of age) of both sexes, referred to a private center from Internal Medicine, Endocrinology, Surgery and Oncology Clinics between February 2017 to October 2019.

Results: Ultrasound Elastography (USE) is a reliable noninvasive diagnostic procedure for evaluating dominant thyroid nodules. According to elastography grade, $60 \%$ nodules were benign and $40 \%$ were malignant. The benign histology of soft nodules on qualitative elastography can be predicted with a satisfactory level of certainty. In cases of hard lesions, the predictive probability for a malignant finding is very high. The semi-quantitative Elasticity Ratio (ER) is a useful index in the differential diagnosis of thyroid nodules since it can provide quantitative information about the stiffness of the nodules however ER ratio calculation did not provide additional data to the qualitatively assessed elastography scores, which still remains the USE reference parameter.

Conclusion: Elastography is undeniably a useful technological advance in imaging of thyroid nodules but as with any other imaging modality it has its drawbacks. To overcome these limitations, it should always be performed by an experienced operator who is familiar with its potential pitfalls and its results should always be interpreted in conjunction with findings of the B-mode sonography.

Key Words: Sono-Elastography - Benign - Malignant thyroid nodules.

\section{Introduction}

THE name thyroid gland, in English, is derived from the Latin glandulathyreoidea, meaning shield-

Correspondence to: Dr. Sara Medhat Mohamed, E-Mail: Doctor.s.15.1.84@ gmail.com shaped gland, named by Thomas Warton in 1656 . As the first endocrine gland to develop embryologically, it possesses important functions from fetal development until death [1].

Nodular thyroid disease is a common finding in the general population, in particular in iodinedeficient areas. Thyroid nodules are palpable in $5 \%$ of people. The majority of thyroid nodules are benign; however, $15-30 \%$ are classified as indeterminate or suspicious for malignancy [2].

The primary challenge in the evaluation of thyroid nodules is to reliably identify the majority of benign nodules that do not require surgical removal, while avoiding the risk that malignant (or pre-malignant) nodules are not identified, so missing an opportunity to provide effective early surgical treatment [3].

Fine-Needle Aspiration (FNA) is the standard procedure to determine whether a thyroid nodule is cancerous. However, FNA is an invasive procedure, and about $10-20 \%$ of FNAs yield inadequate results and lead to repeat biopsy [4].

Thyroid Ultrasound (US) being easily accessible, noninvasive, and cost-effective, is a key examination for the management of thyroid nodules. Thyroid US assessment of the risk of malignancy is crucial in patients with nodules, in order to select those who should have a Fine Needle Aspiration (FNA) biopsy performed. Certain features of thyroid nodules on Ultrasound (US) are predictive of malignancy and are used as criteria for FNA. Ultrasound characteristics that have been reported as potential predictors of thyroid malignancy include irregular margins, hypo-echogenicity, absence of a halo, a predominantly solid composition, or presence of calcification. These criteria have various sensitivity and specificity, but unfortunately 
none of them alone is sufficient to discard or detect malignancy efficiently [5].

Alternative ultrasound techniques are now being used to improve the diagnostic accuracy of ultrasound, as many suspicious ultrasound features can exist in both benign and malignant nodules [6].

Ultrasound elastography, which was introduced in the 1990s, provides real-time information regarding the tissue elasticity and allows in vivo assessment of the tissue's mechanical properties, mapping of tissue stiffness, and characterization of soft tissue lesions. Ultrasound elastography is based on the principle that, under compression, the softer parts of tissues deform easier than the harder parts. Ultrasound Elastography is reported to be useful in differentiation of the benign and malignant lesions of the prostate, breast, pancreas, and lymph nodes. In the last years, important studies have been conducted in the differential diagnosis of thyroid nodules by US elastography [7].

\section{Aim of the work:}

The aim of this study is to assess the diagnostic value of elastography in the evaluation of thyroid nodules.

\section{Patients and Methods}

\section{Patients:}

This study was performed on 25 thyroid nodules in 25 adult patients ( $>18$ years of age) of both sexes, referred to our private center from Internal Medicine, Endocrinology, Surgery and Oncology Clinics between February 2017 to October 2019.

Elastography was performed on the largest suitable nodule in cases of Multinodular Goiter (M.N.G.) and the only nodule in case of solitary thyroid nodule, taking care to ensure that the nodule to be examined fulfilled the following criteria: Adequate amount of adjacent normal thyroid tissue was available for comparison, size of the nodule was more than $5 \mathrm{~mm}$ and predominantly solid nodule with no or small cystic areas.

Patients with isthmic nodules, nodules adjacent to common carotid were avoided, large nodules occupying $>75 \%$ of thyroid lobe volume, nodules with a cystic component $>15 \%$ of the nodule volume and nodules with peripheral egg-shell calcification or nodules with extensive intra-lesional coarse calcifications were excluded from the study.

\section{Patient preparation:}

The examination required no patient preparation. Informed written consent was obtained from all patients and this study was approved by our department and our ethics review committee.

\section{Machine: GE Logic S7 expert.}

\section{Technique and image acquisition:}

For all patients, the US examination was started with B-mode imaging. The study was performed with the patient in the supine position. The transducer was applied to the neck using adequate amount of gel, with hyper-extended neck and the chin was elevated. During B-mode US, thyroid gland lesions were identified and a box was highlighted by the operator to encompass the nodule that fulfilled the above mentioned criteria taking care to include in it as much "normal" reference thyroid tissue as available. Light pressure was generated by the hand held US transducer. The quality of the operator's free-hand pressure was visualized by real-time measurement displayed on a five-point numerical scale, and pressure degree was constantly maintained at level 3-4 throughout the all examinations. Allowing the operator to assess the validity of the compression cycles in real-time. The resultant elastographic image, also known as elastogram, was represented as a color coded image superimposed on the B-mode image and displayed next to it on the screen. Blue represented hard, stiff tissue (with lowest elastic strain or no strain), red represented soft tissue (with greatest elastic strain), and green or orange represented intermediate level of stiffness.

\section{Image analysis:}

Elastograms of nodules were qualitatively evaluated with a stepwise scoring system, according to the prevalent color in the nodule using the 5point classification system described by Rago et al. [8], where a score of 1 defined elasticity that is entirely soft in the nodule, 2 as mostly soft in the nodule, 3 as peripherally soft, 4 as entirely hard in the nodule (no elasticity), and 5 as hard in the nodule and surrounding area of posterior shadowing.

Semiquantitative analysis was based upon generation of the Elasticity Ratio (ER) which compares the stiffness of two different areas within the same image: Two Regions of Interest (ROIs) were manually applied on the screen, one on the nodule and the second on the reference normal thyroid, and the ER was calculated by dividing the elasticity index of the nodule divided by the elasticity index of the reference thyroid tissue. 
Tissue based diagnosis: Final diagnosis of nature of the thyroid nodules was made based upon US guided fine needle aspiration cytology and/or histopathological evaluation of surgical specimens following surgical removal of the nodule.

Statistical analysis: Statistical analysis was performed using MedCalc statistical software for Windows (MedCalc Software, Mariakerke, Belgium). Data for continuous variables were expressed as mean $\pm \mathrm{SD}$, median, interquartile range and range and as both number and percentage for categorical data. Differences in the distribution of categorical variables between groups were evaluated by the 2-tailed Chi-square $\left(\chi^{2}\right)$ test or Fisher exact test. The 2-tailed Student- $t$-test was used to test for statistically significant differences in the quantitative variables between groups. The Receiver Operating Characteristic (ROC) curve analysis was used to identify the optimal cutoff of the quantitative variables according to the Youden index. The diagnostic accuracy of all variables was evaluated in terms of sensitivity, specificity, Positive Predictive Value (PPV), Negative Predictive Value (NPV), and Area Under the ROC curve (AUC). For all tests all $p$-values were two-tailed and a $p$-value $<0.05$ was considered significant.

\section{Results}

A total of 25 thyroid nodules in 25 patients were assessed in this study. 15 patients were diagnosed with benign nodules (adenoma, $\mathrm{n}=10$; nodular goiter, $n=4$; focal thyroiditis, $n=1$ ). 10 patients were diagnosed with malignant nodules (papillary carcinoma, $n=5$; follicular carcinoma, $n=5$ ) Fig. (1). Patients' demographic data can be seen in (Table 1) and Figs. $(2,3)$. No statistically significant difference was seen between the benign and malignant groups as regards the age, sex of patients and the location of thyroid nodules.

Table (1): Demographic characteristics of the studied patients.

\begin{tabular}{|c|c|c|c|c|}
\hline Characteristics & $\begin{array}{c}\text { All } \\
\text { patients }\end{array}$ & Benign & Malignant & $\begin{array}{c}p- \\
\text { value }\end{array}$ \\
\hline \multicolumn{5}{|l|}{ Age: } \\
\hline Mean \pm SD & $41.1 \pm 17.4$ & $39.0 \pm 15.5$ & $44.3 \pm 20.2$ & \multirow{3}{*}{$\begin{array}{l}p^{=} \\
0.4661 \mathrm{a}\end{array}$} \\
\hline Range (min-max) & $18-80$ & $18-66$ & $20-80$ & \\
\hline Median (IQR) & $41(26.8-50.8)$ & $40(28-49)$ & $45(23-53)$ & \\
\hline \multicolumn{5}{|l|}{ Sex, $n(\%):$} \\
\hline Males & $5 \quad(20)$ & $2(13.3)$ & $3(30)$ & \multirow{2}{*}{$\begin{array}{l}p^{=} \\
0.358 \mathrm{~b}\end{array}$} \\
\hline Females & $20(80)$ & $13(86.7)$ & $7(70)$ & \\
\hline \multicolumn{5}{|l|}{ Laterality, $n(\%)$ : } \\
\hline Right & $15(60)$ & $9(60)$ & $6(60)$ & \multirow{2}{*}{$p_{1.000 \mathrm{~b}}^{=}$} \\
\hline Left & $10(40)$ & $6(40)$ & $4(40)$ & \\
\hline
\end{tabular}

$\mathrm{N}$ : Number. IQR : Interquartile Range.

Min : Minimum. a $: p$-value determined by $t$-test

Max : Maximum. $\mathrm{b} \quad: p$-value determined by Fisher's exact test.
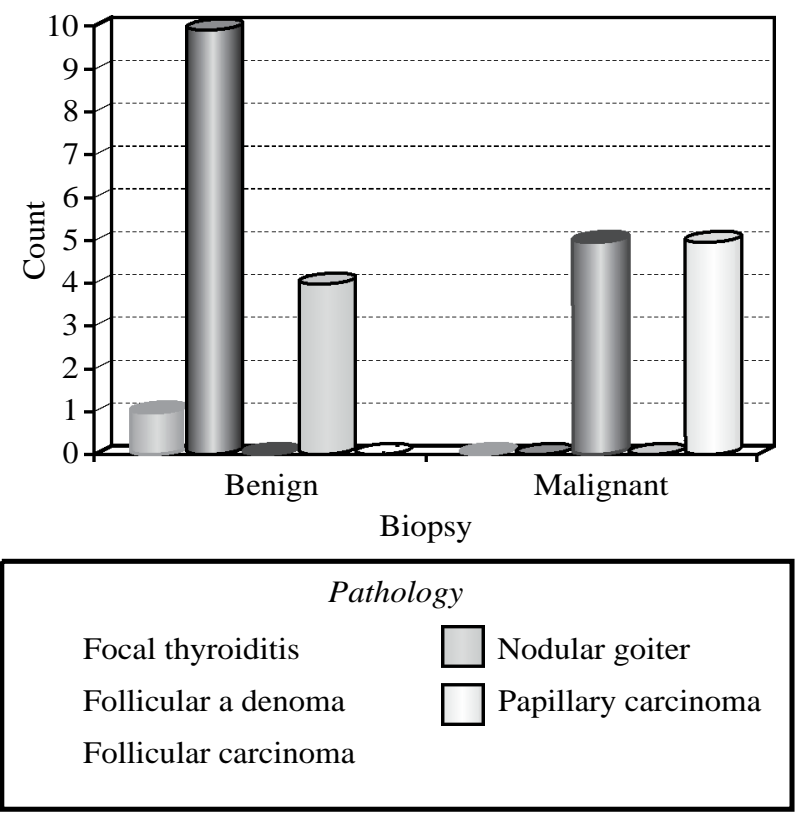

Fig. (1): Results of the histopathological evaluation of the thyroid nodules in benign and malignant groups.

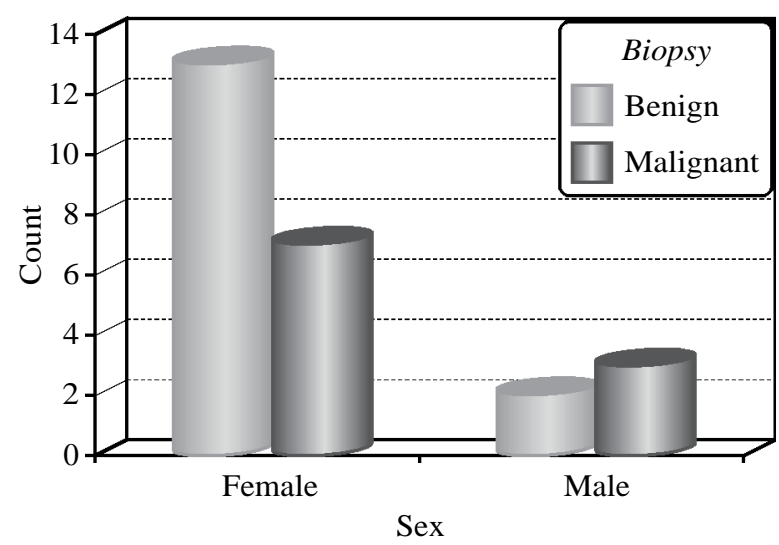

Fig. (2): Number of female and male patients in benign and malignant groups.

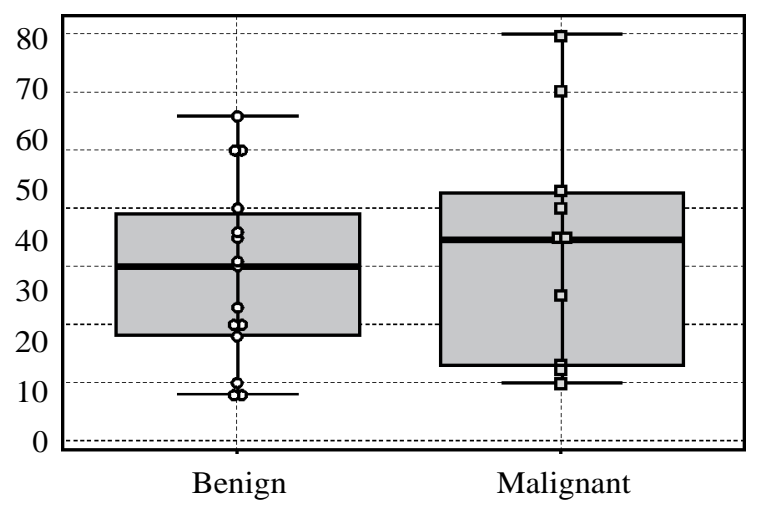

Fig. (3): Box plots for age in the benign and malignant groups. Middle lines represent median values, boxes represent $25^{\text {th }}$ to $75^{\text {th }}$ percentiles, and whiskers demonstrate range.

The distribution of elastography grades between benign and malignant thyroid nodules are seen in (Table 2) and Fig. (4). In total, 15 (100\%) of the 
benign thyroid nodules were classified as grade 1 and 2 , while $7(70 \%)$ of the malignant thyroid nodules were classified as grade 3 and 4 and only $3(30 \%)$ of malignant nodules were classified as grade 2 . No grade 5 nodules were encountered in this study. A higher elastography grade was significantly associated with malignant thyroid nodules and vice versa $\left(\chi^{2}=14.71, p=0.0021\right)$.

Table (2): Distribution of elastography grades among benign and malignant groups.

\begin{tabular}{llll}
$\begin{array}{l}\text { Elastography } \\
\text { grade }\end{array}$ & $\begin{array}{c}\text { Benign, } \\
\mathrm{n}(\%)\end{array}$ & $\begin{array}{c}\text { Malignant, } \\
\mathrm{n}(\%)\end{array}$ & \multicolumn{1}{c}{$\begin{array}{c}\text { Total, } \\
\mathrm{n}(\%)\end{array}$} \\
\hline 1 & $1 / 15(6.6)$ & 0 & $1 / 25(4)$ \\
2 & $14 / 15(93.3)$ & $3 / 10(30)$ & $17 / 25(68)$ \\
3 & 0 & $1 / 10(10)$ & $1 / 25(4)$ \\
4 & 0 & $6 / 10(60)$ & $6 / 25(24)$ \\
5 & 0 & 0 & 0 \\
\hline Total & $15 / 25(60)$ & $10 / 25(40)$ & $25 / 25(100)$ \\
\hline
\end{tabular}

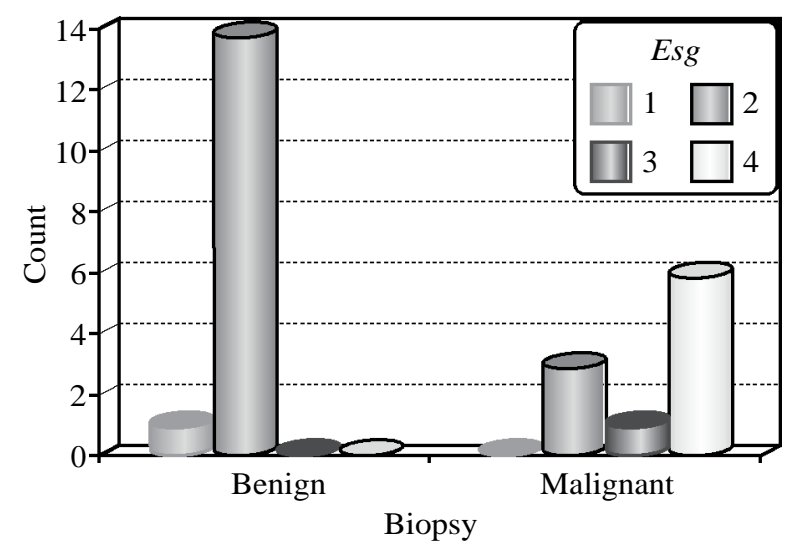

Fig. (4): Bar chart showing the distribution of the different elastography grades between benign and malignant thyroid nodules.

Esg: Elastography Grade.

E ratios of all nodules (benign and malignant) are seen in (Table 3) and Fig. (5). The malignant thyroid nodules exhibited a significantly increased elasticity ratio compared with that of benign nodules $(p=0.0018)$. However, overlap existed in the individual $\mathrm{E}$ ratios among both groups.

Table (3): Eratiosin benign and malignant groups.

\begin{tabular}{lllll}
\hline E ratio & $\begin{array}{c}\text { All } \\
\text { patients }\end{array}$ & Benign & Malignant & $\begin{array}{c}p \text { - } \\
\text { value }\end{array}$ \\
\hline Mean \pm SD & $2.86 \pm 1.26$ & $2.26 \pm 0.99$ & $3.75 \pm 1.11$ & $p=$ \\
Range (min-max) & $0.6-5.1$ & $0.6-4$ & $2.1-5.10$ & $0.0018 \mathrm{a}$ \\
Median (IQR) & 2.80 & 2.25 & 3.97 & \\
& $(2.03-3.89)$ & $(1.72-2.95)$ & $(3-4.85)$ & \\
\hline
\end{tabular}

E-ratio : Elasticity ratio.

IQR : Interquartile Range.

a $\quad: p$-value determined by $t$-test.

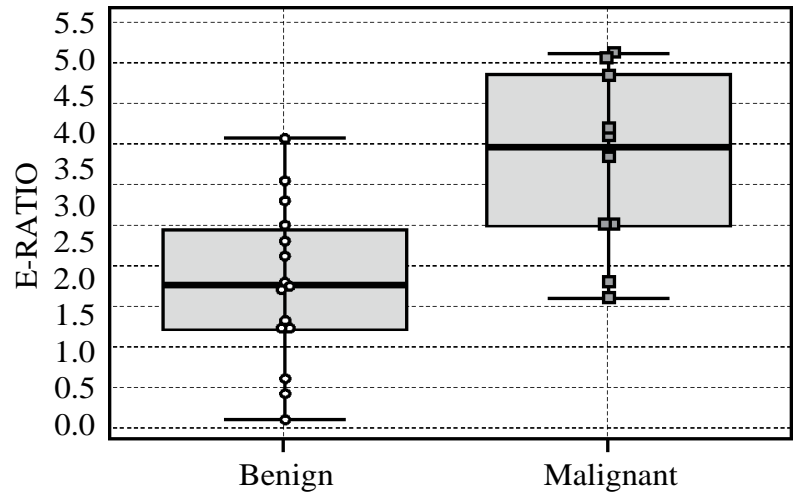

Fig. (5): Box plots for elasticity ratio in the benign and malignant groups. Middle lines represent median values, boxes represent $25^{\text {th }}$ to $75^{\text {th }}$ percentiles, and whiskers demonstrate range.

Since elastography grade and elasticity ratio demonstrated significant differences between benign and malignant thyroid nodules, additional ROC curve analysis was performed to compare the discriminating ability between these two diagnostic parameters Fig. (6). No significant difference was identified for AUC between elastography grade and elasticity ratio $(p=0.864)$. Furthermore, malignant thyroid nodules could be differentiated from benign nodules using a cutoff elastography grade of $>2$ and a cutoff elasticity ratio of $>2.8$ with sensitivities, specificities, PPVs, NPVs of $70 \%$, $100 \%, 100 \%, 83.3 \%$ and $80 \%, 73.3 \%, 66.7,84.6 \%$ respectively.

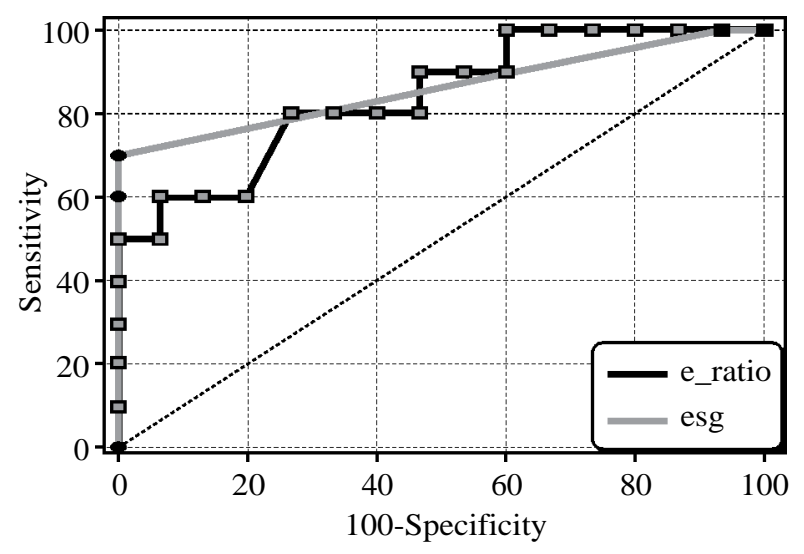

Fig. (6): Receiver operating characteristic curve analyses. The specificity and sensitivity of using elastography grade and elasticity ratio were compared. The AUC was 0.860 (95\% CI, 0.719-1.000), 0.840 (95\% CI, $0.678-1.000)$ for elastography grade and elasticity ratio respectively. However, no significant difference in AUC was identified between elastography grade and elasticity ratio $(p=0.864)$.

AUC: Area Under the Curve.
CI : Confidence Interval.

There was no statistically significant difference in the frequency of occurrence of the different 
elastography grades among follicular and papillary carcinoma subgroups $\left(\chi^{2}=1.33, p=0.513\right)$ (Table 4).

Table (4): Distribution of elastography grades in follicular and papillary carcinomas subgroups.

\begin{tabular}{llll}
\hline $\begin{array}{l}\text { Elastography } \\
\text { grade }\end{array}$ & $\begin{array}{c}\text { Papillary C, } \\
\mathrm{n}(\%)\end{array}$ & $\begin{array}{c}\text { Follicular C, } \\
\mathrm{n}(\%)\end{array}$ & $\begin{array}{c}\text { Total, } \\
\mathrm{n}(\%)\end{array}$ \\
\hline 1 & 0 & 0 & 0 \\
2 & $1 / 5(20)$ & $2 / 5(40)$ & $3 / 10(30)$ \\
3 & $1 / 5(20)$ & 0 & $1 / 10(10)$ \\
4 & $3 / 5(60)$ & $3 / 5(60)$ & $6 / 10(60)$ \\
5 & 0 & 0 & 0 \\
\hline Total & $5 / 10(50)$ & $5 / 10(50)$ & $10 / 10(100)$ \\
\hline
\end{tabular}

A statistically significant difference was found between the $\mathrm{E}$ ratios of papillary and follicular carcinomas $(p=0.0402)$ (Table 5).

Table (5): E ratios in papillary and follicular carcinoma groups.

\begin{tabular}{llll}
\hline E ratio & \multicolumn{1}{c}{ Papillary C } & Follicular C & $p$-value \\
\hline Mean \pm SD & $3.07 \pm 0.89$ & $4.44 \pm 0.88$ & $p=$ \\
Range (min-max) & $2.10-4.10$ & $3.0-5.10$ & $0.0402 \mathrm{a}$ \\
Median (IQR) & $3.0(2.24-3.91)$ & $4.85(3.9-5.06)$ &
\end{tabular}

\begin{tabular}{ll}
\hline E-ratio: Elasticity ratio. & IQR: Interquartile Range. \\
a $\quad: p$-value determined by $t$-test. &
\end{tabular}

- Clinical background:

Female patient 28 years' old, with an accidentally discovered thyroid nodule.

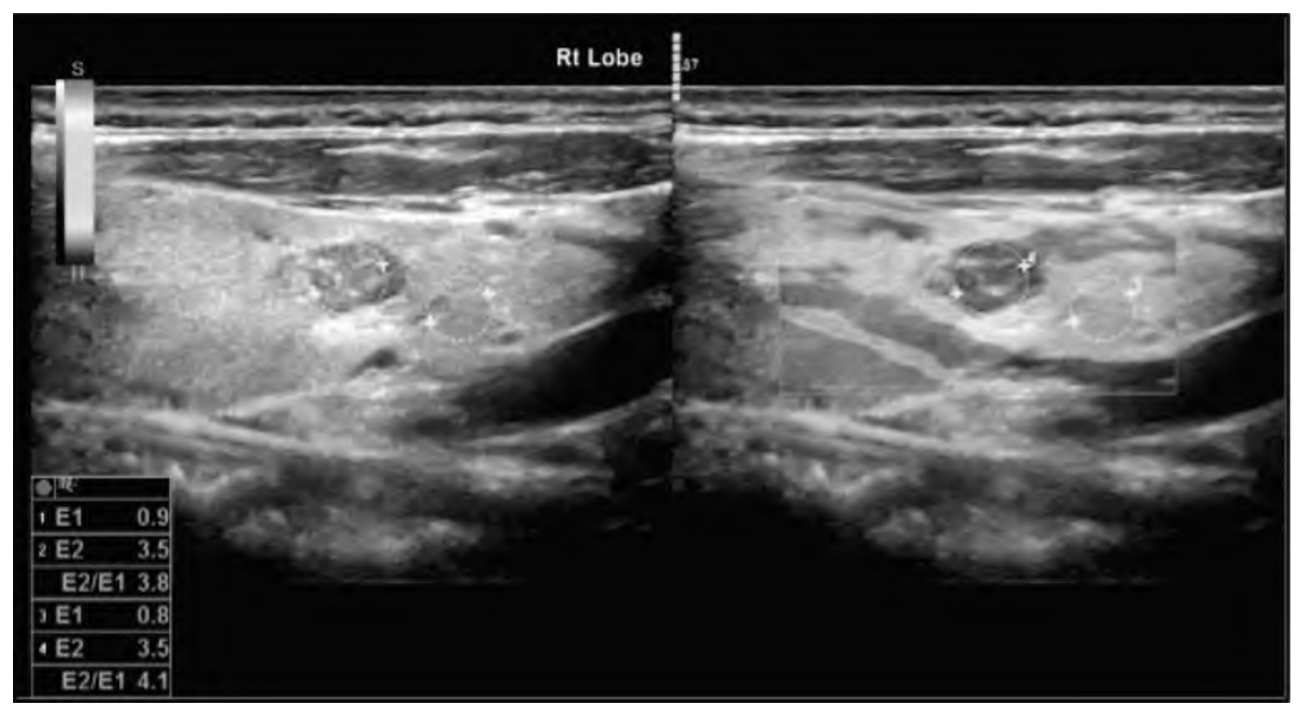

Fig. (7): Grayscale sonographic map (left) and elastographic map of a right thyroid lobe nodule. B-mode U/S shows a hypoechoic nodule with well-defined margins and punctate microcalcifications. The corresponding elastogram shows a nodule with limited elasticity that is decidedly stiff compared with the surrounding thyroid tissue (grade 4 ). The elasticity ratio was 4.1. Histopathology confirmed the presence of papillary thyroid carcinoma.

\section{Discussion}

Thyroid nodules, with their high prevalence in the general population, represent a diagnostic challenge for clinicians. Ultrasound (US), although absolutely reliable in detecting thyroid nodules, is still not accurate enough to differentiate them into benign and malignant. As thyroid cancers have a harder consistency than benign thyroid nodules so Ultrasound Elastography (USE), has been introduced in order to further increase US accuracy and aiming reduction of thyroid biopsies. USE is an US-based technique to assess the biomechanical properties of tissue in the clinical setting. It is based upon the principle that, under compression, the softer parts of tissues deform easier than the harder parts; providing an estimation of tissue stiffness through comparing the stiffness of benign and malignant tumors with the stiffness of the surrounding normal parenchyma. By use of this technique, two types of elasticity assessment can be acquired: (I) Visual scoring measured by means of colors within the nodule, (II) Semi quantitative measurement of the elasticity index or strain ratio [9].

Results of the current study showed that qualitative elastography grades were able to differentiate benign and malignant thyroid nodules where higher Elastography Grades (ESGs) were more frequent in malignant nodules as opposed to benign nodules which consistently displayed lower elastography grades. In this study, a cutoff elastography grade of $>2$ could distinguish malignant thyroid nodules 
from benign nodules with a sensitivity, specificity, PPV, NPV of $70 \%, 100 \%, 100 \%, 83.3 \%$ respectively. Results of this study were compatible with many previous studies regardless of whether a 4 or a 5 point scoring system was used. Esfahanian et al., [10] reported that ESGs were significantly higher in malignant nodules and that the best point to differentiate benign and malignant thyroid nodules was 2 with sensitivity and specificity of $61 \%$ and $78 \%$. Asteria et al., [11] evaluated 86 nodules in 66 patients. They reported the cut-off point between 2 and 3 as the best cut-off point to differentiate benign and malignant thyroid nodules with sensitivity and specificity as $94 \%$ and $81 \%$. Rago et al., [8] applied 5 scale scoring system and found that ESGs 4-5 were highly predictive of malignancy with sensitivity and specificity of $97 \%$ and $100 \%$. Similarly, Gietka-Czernel et al., [12] used 5 scale scoring to differentiate benign and malignant thyroid nodules in 52 patients and demonstrated that ESGs of 4 or 5 were highly predictive of malignancy with sensitivity and specificity of $86 \%$ and $97 \%$. Concordant results were also reported by Friedrich-Rust et al., [13], Wang et al., [14] and Shao et al., [15]. It is important to note that despite the consistent pattern of results described by these studies the calculated sensitivities, specificities, PPVs and NPVs differed considerably among the different studies including this study. This variation in values could be explained by differences in the inclusion criteria and the sample sizes used in different studies.

In spite of these promising results, overlap exists between the ESGs of benign and malignant nodules in individual cases. While no benign nodules displayed an ESG more than 2 in this study, $30 \%$ of the malignant nodules displayed an elastography grade of 2 . Likewise, all of the abovereferenced studies showed that with intermediate ESGs of 2 and 3 and occasionally 4, misclassification of benign and malignant nodules may be unavoidable in individual cases. In some studies, the overlap was so great that it led the authors to question the value of USE. Lippolis et al., [16] reported that malignancy was detected in $50 \%$ of the nodules with ESG of 1-2 and in 34\% of those with grades 3-4. Kagoya et al., [17] found that only 9 out of 21 thyroid nodules with ESGs of 3-4 were malignant, and though they found that patterns 3 and 4 of elastogram scan could be considered as prognostic values of malignancy, their sensitivity and specificity were diminished. Moon et al., [18] and Zhang et al., [19] argued that USE was not as useful as it was once thought to be with a diagnostic performance inferior to that of conventional ultra- sonography in differentiation of benign and malignant thyroid nodules.

Several factors influence the diagnostic performance of USE and can explain the reasons behind the encountered false results, the most important of which is the histopathological type of the thyroid nodule. Papillary thyroid carcinoma, the most common type of thyroid cancer, is characterized by complex papillae with a central fibrovascular stalk, usually accompanied by psammoma bodies and fibrosis making them relatively hard [15]. In this study, only 1 out of a total of 5 papillary carcinomas was one of the 3 malignant lesions which displayed an ESG of 2. Although uncommon, papillary carcinomas misdiagnosed as benign lesions on the basis of USE were also reported by several studies including those by Sebag et al., [20], Bojunga et al., [21], Moon et al., [18] and Shao et al., [15]. The existence of microcystic areas of degeneration and different histological variants of papillary carcinoma, some of which are softer than others may explain these confounding findings $[15,20]$. Two out of the $5(40 \%)$ follicular carcinomas in this study accounted for the majority $(2 / 3)$ of the malignant lesions that demonstrated an ESG of 2. Though our sample size was small, this result indicated that follicular carcinomas could be easily diagnosed as benign nodules as opposed to papillary carcinomas which are more frequently hard lesions. Similar findings were highlighted by Shao et al., [15], Bojunga et al., [21] and Hong et al., [22]. The gross anatomy and cellular patterns of follicular carcinoma overlap with those of benign follicular adenoma; both are composed of small microfollicles with variable amounts of colloid. Indeed, follicular carcinoma could be differentiated from benign follicular adenoma only when a capsular or vascular invasion was discovered upon histological examination and hence, it would be expected that their elasticity patterns won't differ much from their benign counterparts, making them more susceptible to misdiagnosis as benign lesions [15]. Nevertheless, some follicular carcinomas display increased cellular content which makes them stiff enough to be correctly diagnosed by elastography [22]. $60 \%$ of the follicular carcinomas displayed an ESG of 4 in this study. Finally, though unencountered in this study, thyroid lymphoma, medullary carcinoma and undifferentiated carcinoma were reported to demonstrate low stiffness and were misdiagnosed as benign lesions by USE [23,24].

The effect of size of the thyroid nodules on the diagnostic performance of USE was not investigated in this study, however Hong et al., [22] and Moon et al., [18] reported diminished diagnostic 
accuracy of USE in the detection of malignancy in nodules with a greatest diameter of $1 \mathrm{~cm}$ or less. They reported that this may have been due to nonaxial and out-of-plane motion of the nodule during freehand external compression. Controversially, Rago et al., [8] reported that the performance of USE was independent of the nodular size. Given that nodular size may be a potential cause of false results, larger studies aimed at investigating the reliability of USE in correctly diagnosing thyroid nodules less than $1 \mathrm{~cm}$ in size are indicated.

Nodule position is another factor which can influence the results of elastography. During elastography, by comparing echoes made with compression, one can obtain information about how hard or soft the tissues are relative to their surroundings and thus if a nodule was not entirely surrounded by thyroid tissue this would alter its elastographic pattern giving false results. Therefore, anterior nodules, protruding to the capsule, maybe mislabeled as soft because the reference tissue is represented by strap muscles, not by the thyroid parenchyma. Isthmic nodules are also difficult to assess, being compressed between two hard planes (transducer and trachea) and lacking reference tissue. Deep nodules are subject to the stress decay phenomenon because stress transmission is reduced as the distance from the transducer increases. Less tissue dislocation in deep portions of the thyroid will induce artefactual hardening [24]. This was elegantly depicted by Hong et al., [22] where 2 papillary thyroid carcinomas (greatest diameter, 7 and $13 \mathrm{~mm}$ ) protruded from the thyroid anterior capsule, and they both had a score of 2. Alternatively, two other hyperplastic nodules (benign, greatest diameter, 29 and $32 \mathrm{~mm}$ ) protruded from the thyroid posterior capsule and extended to the posterior sternum. They both had a score of 4 . Furthermore, the nodules located in front of the common carotid artery are the most susceptible to pulsation interferences increasing the liability for false results [24]. Other than excluding isthmic nodules and those adjacent to the carotid, we did take into consideration the exact position of the misdiagnosed nodules and the potential contribution of the location of the nodules to the performance of USE these findings cannot be verified by this study, yet we recognize that this a potential cause of false results in this study.

Lastly, since elastograms of thyroid nodules give information on how elastic or stiff a thyroid nodule is in comparison to the surrounding tissue, the presence of a sufficient amount of healthy thyroid tissue is an essential pre-requisite for accurate results. If the surrounding thyroid tissue was afflicted by a diffuse disease such as thyroiditis which would subsequently affect the elastic properties of the thyroid tissue, or in cases of insufficient healthy tissue as in cases of extensive MNG, elastograms of any superimposed nodules would be altered giving rise to potentially false and cofounding results [22]. Fortunately, this was not an issue in this study as none of the cases of nodular goiter were extensive enough to hinder USE (due to our strict inclusion criteria) and the single case of thyroiditis was correctly diagnosed as benign by USE, nevertheless, since it is a potential cause of false results, it is a point worth mentioning.

A final note to make regarding the diagnostic performance of the qualitative results of USE in this study is that Rago's score 2 describes a mixed elastographic pattern with areas of green and blue, yet it does make a distinction based upon the amount of blue or green areas in the mixed pattern. This means that as long as a mixed pattern is seen, harder lesions with predominantly blue areas and softer lesions with predominantly green areas were both given a score of 2 which might impair diagnostic accuracy. Thus, using a 6 point scoring system such as that described by Hong et al., [22], where an additional grade ofelasticity describing a nodule which is mostly stiff save for small spots of higher elasticity was added to the Rago criteria, may potentially allow more accurate segregation of benign and malignant nodules. Further studies aimed at defining the most accurate scoring system is necessary, as currently the proposed systems of scoring the elastography image of thyroid nodules vary from 6 to 2 patterns, each of them with variants and different reported diagnostic values as described in the review by Dudea and Botar-Jid [24].

In addition to the qualitative evaluation of strain elastograms, semi-quantitative evaluation can also be performed by the generation of Strain Ratios (SR). Strain is a value which represents the relative deformation of a structure under pressure. Calculation of SR implies placement of two similarsized ROIs, at similar depth from the transducer (depth difference should be less than $10 \mathrm{~mm}$ ), one in the nodule (A) and the second in neighbouring normal parenchyma (B). SR is then computed as B/A. Alternatively, some US vendors like General Electric, which was used in this study, generate elasticity ratios rather than strain ratios. The Elasticity Index (EI) is a value between 0 (softest) to 6 (hardest) which represents the relative hardness or stiffness of a structure within an elastographic image [24]. Similar to the technique employed in calculation of strain ratios, generation of the Elasticity Ratio (ER) involves defining 2 ROIs, one in 
the nodule (A) and the other in the adjacent normal thyroid tissue (B), however, ER is calculated as the EI of the nodule divided by the EI of the reference thyroid tissue (A/B) [25]. Malignant nodules are harder than benign nodules and thus have lower strain values and higher elasticity indices compared to benign nodules. Accordingly, and based upon the computational methods described above, the likelihood of malignancy increases with an increase in strain ratio or an increase in elasticity ratio.

The vast majority of the published studies use SR and all have reported that the SRs in malignant nodules were significantly higher than their benign counterparts however there was no consensus regarding the optimal cutoff value for differentiation of malignant and benign nodules [24] lists the diagnostic performance of SR in the differentiation of thyroid nodules described by a number of older studies.

There was also no agreement between the various studies on whether qualitative elastographyscores or semi-quantitative SRs were better in distinguishing benign and malignant thyroid nodules. While Xing et al., [23] and Razavi et al., [26] reported better performance for SR that the elastography scores, Chong et al., [27] found that adding SR to the color scale classification does not improve the diagnosis, on the contrary, the association of the two was found to lead to lower diagnostic efficacy. Friedrich-Rust et al., [28] reported that no significant difference was observed between semiquantitative and qualitative analysis.

Unfortunately, the number of studies using the unpopular approach where the stiffness value (EI) of the nodule is divided by the stiffness value of neighboring thyroid is very limited. Guazzaroni et al., [29] found that malignant thyroid nodules were associated with higher stiffness ratios and a cutoff value greater than 3.16 had associated sensitivity of $92.3 \%$, specificity of $87.4 \%$, positive and negative predictive values of $54.5 \%$ and $98.6 \%$ respectively in distinguishing malignant from benign nodules. In this study, malignant thyroid nodules could be differentiated from benign nodules using a comparable cutoff elasticity ratio of $>2.8$ with a sensitivity, specificity, PPV, NPV of $80 \%, 73.3 \%$, $66.7,84.6 \%$ respectively. This mild discrepancy in the reported values for the diagnostic performance is likely related to differences in the study population and size. Guazzaroni et al., [29], however, found quantitative analysis to provide no supplemental information, compared to the qualitative elasticity scores. And similarly, we found no added value to or improved diagnostic performance of the semiquantitative ratios when compared to the qualitative analysis.

As can be seen, all studies assessing the diagnostic performance of the semi quantitative measurements of USE, including this study reported the presence of false-negative and false-positive results. In this study, a cutoff value of 2.8 , falsely diagnosed 2 papillary carcinomas as benign and misdiagnosed 2 cases of MNG and follicular adenoma as malignant. Causes of false results for semiquantitative analysis are numerous and are mostly similar to the ones described for the elastography scores. Among the possible causes which may have yielded false measurements in this study is the possibility of sampling error. As reported by Cantisani et al., [30], the whole nodule should be encompassed in the measurement area, and this was sometimes difficult, due to irregular shape and unclear margins.

Furthermore, in large nodules, non-uniform nodule compression, areas with alternating stiffness as well as the lack of comparable size reference tissue may have led to confusing results as was described by Stoian et al., [31]. Transverse scans through the thyroid lobe are more susceptible tointerference with carotid pulsations, therefore the longitudinal scans are preferred by several authors including Xing et al., [23] and Ning et al., [32]. Conversely, Friedrich-rust et al., [28] supported taking the best access, as was undertaken in this study, since no significant difference was observed between the results obtained in the transverse and longitudinal positions. For the calculation of semi quantitative indices, the ROI size was adapted to the size of the nodule to obtain an overall elasticity value of the nodule in this study. However, the size of the ROI might influence the elasticity results as explained by Friedrich-Rust et al. [28]. Tissue histology may also explain the false results. The presence of intralesional micro calcifications and fibrosis, as well as atypical adenomas, may explain the falsely high ER encountered in 4 of the benign nodules in this study. Similar findings were described by Xing et al., [23] and Wang et al., [33]. As described before the effect of nodular size on USE findings were not considered in this study but just like with elastography scores, small-sized carcinomas may yield falsely low stiffness values [32]. Other causes which might explain our falsenegative results are the presence of a very well differentiated cancer and the presence of necrosis and tissue softening even in the absence of frank liquefaction [3]. 
This study included 5 cases of papillary carcinoma and 5 cases of follicular carcinoma. As described earlier $2 / 5$ of the follicular carcinoma, as opposed to a single case of papillary carcinoma, had an elastography grade of 2 indicating a tendency for follicular cancers to be softer, even though the difference in frequency of the elastography grades among the two groups did not reach statistical significance. A large number of previous studies described the increased frequency of falsely diagnosed follicular cancers as benign lesions by USE due to their increased softness. In a metaanalysis of eight studies by Bojunga et al., [21], nine follicular carcinomas were reported but four of them were not diagnosed as follicular carcinoma by qualitative USE. Zhang et al., [19] reported that 2 out of 3 follicular carcinomas in their study were misdiagnosed as benign lesions. Controversially, when elasticity ratios were compared between the two groups in this study, the follicular carcinoma group showed a higher elasticity ratio than the papillary carcinoma group, although the statistical significance was slight. Reasons behind why some follicular and papillary cancers can be harder or softer than usual, respectively, have been detailed above. Nevertheless, it must be mentioned that limited or no information is available on the SR and ER values for follicular cancer in the literature, our sample size was too small, and extensive overlap was seen among the individual ER values of both groups. Larger dedicated studies involving both qualitative and quantitative analysis are mandatory before reliable consistent conclusions can be made.

This study had several limitations. First, this study did not evaluate the performance of US elastography in comparison to conventional sonographic criteria, nor did it evaluate the performance of a combination of grey-scale and elastographic criteria which is more realistic as elastography is never used alone in the characterization of thyroid nodules in clinical practice. Second, this study included a relatively small number of cases, and elasticity ratios need to be confirmed in further studies with larger samples. Third, complex and partially cystic lesions were not included in this study, and the application of elastography in those types of lesions requires future studies. Also further large studies are required to investigate how to evaluate the USE images of nodules complicated by diffuse thyroid disease, which was not encountered in this study. Lastly, histopathological evaluation of surgical specimens wasn't available for all thyroid nodules in the study, in which cases, the final diagnosis was made based on the results of
F.N.A.C., and false cytological results are known to exist.

\section{Conclusion:}

Elastography is undeniably a useful technological advance in imaging of thyroid nodules but as with any other imaging modality it has its drawbacks. To overcome these limitations, it should always be performed by an experienced operator who is familiar with its potential pitfalls and its results should always be interpreted in conjunction with findings of the B-mode sonography.

\section{References}

1- GHARIB H.: Thyroid Nodules diagnosis and management. $1^{\text {st }}$ ed. Springer International publishing, 2018.

2- VORLÄNDER C., WOLFF J., SAALABIAN S., et al.: Real-time ultrasound elastography-a noninvasive diagnostic procedure for evaluating dominant thyroid nodules. Lange becks Arch. Surg., 395: 865-71, 2010.

3- LYSHCHIK A., HIGASHI T., ASATO R., et al.: Thyroid gland tumor diagnosis at US elastography. Radiology, 237: 202-11, 2005.

4- WU H.X., ZHANG B.J., WANG J., et al.: Conventional Ultrasonography and Real Time Ultrasound Elastography in the Differential Diagnosis of Degenerating Cystic Thyroid Nodules Mimicking Malignancy and Papillary Thyroid Carcinomas. Asian pacific journal of cancer prevention, 14, 935, 2013.

5- RUSS G., BONNEMA J., FAIK M., et al.: European Thyroid Association Guidelines for Ultrasound Malignancy Risk Stratification of Thyroid Nodules in Adults: The EU-TIRADS Eur. Thyroid. J., 6: 225-37, 2017.

6- WIENKE J.R., CHONG W.K., FIELDING J.R., et al.: Sonographic features of benign thyroid nodules: Interobserver reliability and overlap with malignancy J. Ultrasound Med., 22: pp. 1027-31, 2003.

7- CANTISANI V., MACERONI P., D'ANDREA V., et al.: Strain ratio ultrasound elastography increases the accuracy of colour-Doppler ultrasound in the evaluation European Radiology, 26 pp. 1441-9, 2016.

8- RAGO T., SANTINI F., SCUTARI M., et al.: "Elastography: New developments in ultrasound for predicting malignancy in thyroid nodules." The Journal of clinical endocrinology and metabolism, 92 (8): 2917-22, 2007.

9- SCHENKE S. and ZIMNY M.: Combination of Sonoelastography and TIRADS for the Diagnostic Assessment of Thyroid Nodules. Ultrasound in Medicine \& Biology, 44 (3): 575-83, 2018.

10- ESFAHANIAN F., ARYAN A., GHAJARZADEH M., et al.: Application of sonoelastography in differential diagnosis of benign and malignant thyroid nodules. Int. J. Prev. Med., 7: 55, 2016.

11- ASTERIA C., GIOVANARDI A., PIZZOCARO A., et al.: US-elastography in the differential diagnosis of benign and malignant thyroid nodules. Thyroid, 18: 523-31, 2008.

12- GIETKA-CZERNEL M., KOCHMAN M., BUJALSKA $\mathrm{K}$., et al.: Real-time ultrasound elastography-A new tool 
for diagnosing thyroid nodules. Endokrynol. Pol., 1: 6527, 2010.

13- FRIEDRICH-RUST M., SPERBER A., HOLZER K., et al.: Real-time elastography and contrast-enhanced ultrasound for the assessment of thyroid nodules. Exp. Clin. Endocrinol. Diabetes, 118: 602-9, 2010.

14- WANG H.L., ZHANG S., XIN X.J., et al.: Application of real-time ultrasound elastography in diagnosing benign and malignant thyroid solid nodules. Cancer Biol. Med., 9: 124-7, 2012.

15- SHAO J., SHEN Y. and LÜ J.: Ultrasound scoring in combination with ultrasound elastography for differentiating benign and malignant thyroid nodules. Clin. Endocrinol (Oxf), 83: 254-60, 2015.

16- LIPPOLIS P.V., TOGNINI S., MATERAZZI G., et al.: Is elastography actually useful in the presurgical selection of thyroid nodules with indeterminate cytology? J. Clin. Endocrinol. Metab., 96: E1826-E1830, 2011.

17- KAGOYA R., MONOBE H. and TOJIMA H.: Utility of elastography for differential diagnosis of benign and malignant thyroid nodules. Otolaryngol. Head Neck Surg., 143: 230-4, 2010.

18- MOON H.J., SUNG J.M., KIM E.K., et al.: Diagnostic performance of gray-scale US and elastography in solid thyroid nodules. Radiology, 262: 1002-13, 2012.

19- ZHANG Y.Z., XU T., GONG H.Y., et al.: Application of high-resolution ultrasound, realtime elastography, and contrast-enhanced ultrasound in differentiating solid thyroid nodules. Medicine (Baltimore), 95: e5329, 2016.

20- SEBAG F., VAILLANT-LOMBARD J., BERBIS J., et al.: Shear wave elastography: A new ultrasound imaging mode for the differential diagnosis of benign and malignant thyroid nodules. J. Clin. Endocrinol. Metab., 95: 5281$5258,2010$.

21- BOJUNGA J., HERRMANN E., MEYER G., et al.: Realtime elastography for the differentiation of benign and malignant thyroid nodules: A meta-analysis. Thyroid, 20: 1145-50, 2010.

22- HONG Y., LIU X., LI Z., et al.: Real-time ultrasound elastography in the differential diagnosis of benign and malignant thyroid nodules. J. Ultrasound Med., 28 (7): 861-7, 2009.
23- XING P., WU L., ZHANG C., et al.: Differentiation of benign from malignant thyroid lesions. Calculation of the strain ratio on thyroid sonoelastography. J. Ultrasound Med., 30: 663-9, 2011.

24- DUDEA S.M. and BOTAR-JID C.: "Ultrasound elastography in thyroid disease". Med. Ultrason., 17 (1): 74-96, 2015.

25- IMAIZUMI A., SASAKI Y., SAKAMOTO J., et al.: Effects of compression force on elasticity index and elasticity ratio in ultrasound elastography. Dentomaxillofacial Radiol., 43 (4): 20130392, 2014.

26- RAZAVI S.A., HADDUCK T.A., SADIGH G., et al.: Comparative effectiveness of elastographic and B-mode ultrasound criteria for diagnostic discrimination of thyroid nodules: A meta-analysis. Am. J. Roentgenol., 200: 131726, 2013.

27- CHONG Y., SHIN J.H., KO E.S., et al.: Ultrasonographic elastography of thyroid nodules: Is adding strain ratio to colour mapping better? Clin. Radiol., 68: 1241-6, 2013.

28- FRIEDRICH-RUST M., VORLAENDER C., DIETRICH C.F., et al.: Evaluation of strain elastography for differentiation of thyroid nodules: Results of a prospective DEGUM multicenter study. Ultraschall Med., 37: 26270, 2016.

29- GUAZZARONI M., SPINELLI A., COCO I., et al.: Value of strain-ratio on thyroid real-time sonoelastography. Radiol. Med., 119: 149-55, 2014.

30- CANTISANI V., LODISE P., GRAZHDANI H., et al.: Ultrasound elastography in the evaluation of thyroid pathology. Current status. Eur. J. Radiol., 83: 420-8, 2014.

31- STOIAN D., CORNIANUZ M., DOBRESCU A., et al.: Nodular thyroid cancer. Diagnostic value of real time elastography. Chirurgia (Bucur), 107: 39-46, 2012.

32- NING C.P., JIANG S.Q., ZHANG T., et al.: The value of strain ratio in differential diagnosis of thyroid solid nodules. Eur. J. Radiol., 81: 286-91, 2012.

33- WANG Y., DAN H.J., DAN H.Y., et al.: Differential diagnosis of small single solid thyroid nodules using realtime ultrasound elastography. J. Int. Med. Res., 38: 466e72, 2010. 


\section{دورتخطيط المرونة بالموجات الصوتية المجية

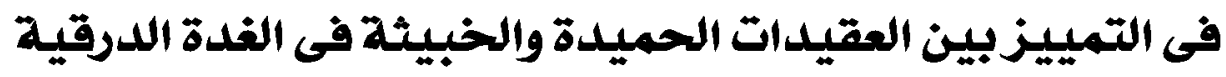

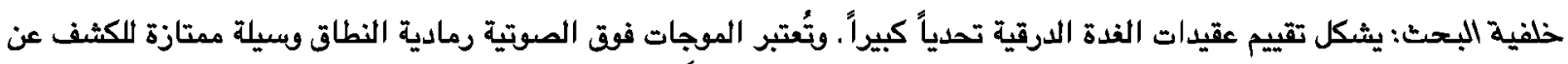

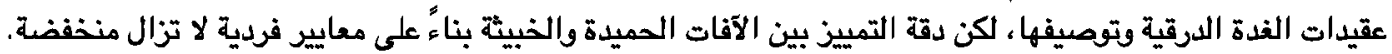

الهدف من الدراسة: تقييم القيمة التشخيصية لتخطيط المرونة بالموجات فوق الصوتية فى تقييم الغدد الدرقية.

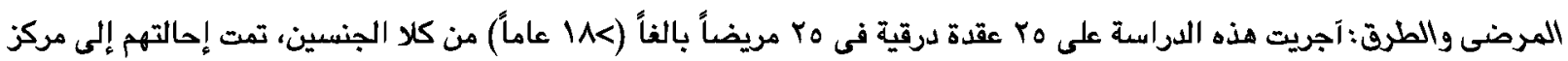

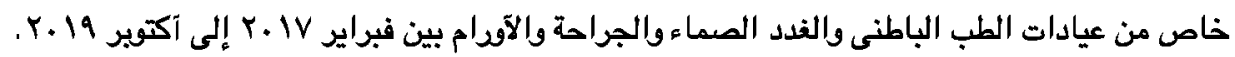

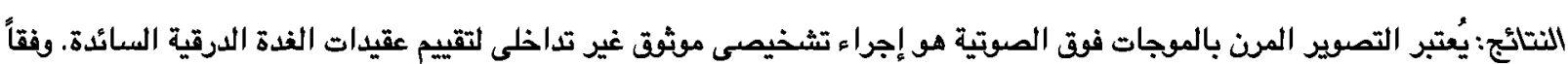

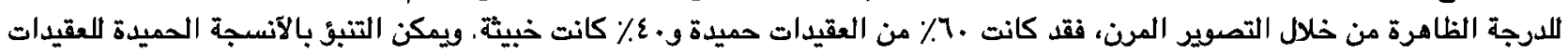

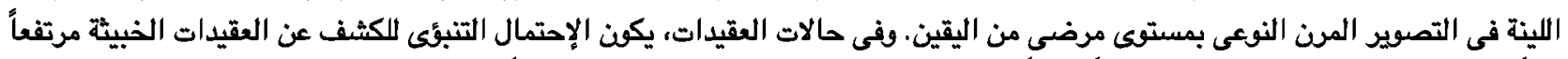

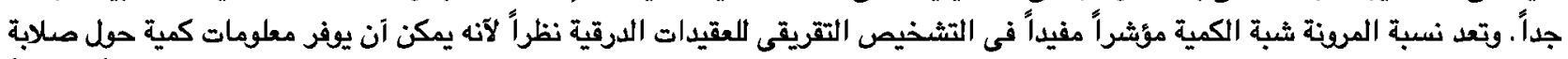

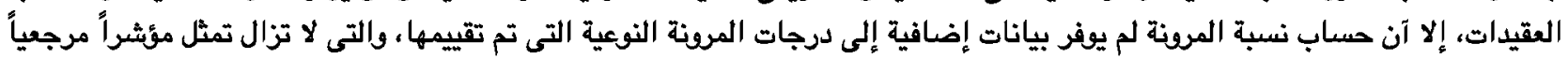

لتخطيط المرونة بالموجات الصوتية.

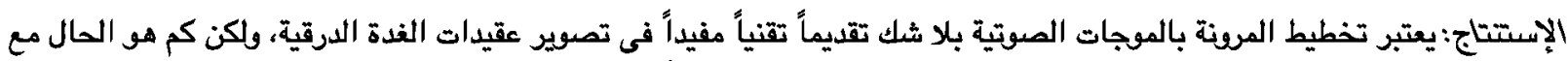

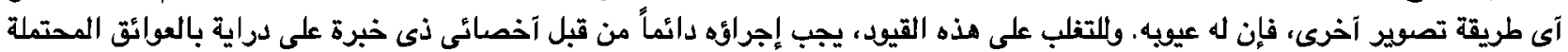

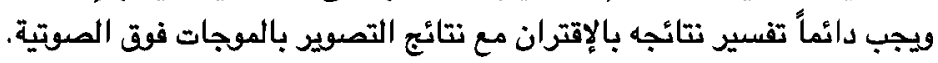

